Supporting Information

\title{
Exploiting Materials to their Full Potential, a Li-ion Battery Electrode Formulation Optimization Study
}

Olivier Rynne ${ }^{1}$, Matthieu Dubarry*2, Corentin Molson ${ }^{1}$, Eva Nicolas ${ }^{1}$, David Lepage ${ }^{1}$, Arnaud Prébé $^{3}$, David Aymé-Perrot ${ }^{4}$, Dominic Rochefort ${ }^{1}$, Mickael Dollé*1

1: Department of Chemistry, Montreal, Université de Montréal, QC H3T1J4, Canada

2: Hawaii Natural Energy Institute, University of Hawai’i at Manoa, Honolulu, HI 96822, USA

3: Hutchinson, 3600 boulevard du Tricentenaire, Montréal, QC H1B 5M8, Canada

4: Total SA, 92069 Paris La Défense, France

*1: Mickael Dollé - mickael.dolle@umontreal.ca

${ }^{* 2}$ : Matthieu Dubarry - matthieu@hawaii.edu

KEYWORDS: design of experiments, formulation, li-ion battery, electrode, optimization 
Analyzing the tortuosity measurements

Three EIS measurements that were carried out on LFP-RUN26 are shown in Figure S2. The RHFR were worth 132,147 and $149 \Omega$, and $Z_{\text {El }}$ were worth 172,171 and $168 \Omega$. Given the ionic conductivity of the electrolyte at $430 \mu \mathrm{S} . \mathrm{cm}^{-1}$, the average tortuosity of LFP-RUN26 was 3.4, with a standard deviation of 1.0. The resulting Mc Mullin number $(\mathrm{Nm})$ was thus equal to 8.0.

\section{Reading ternary diagrams}

The evolution of a response with the variations of 4 distinct parameters could be represented in a regular tetrahedron. However, this option is not suitable for a two dimensional medium, so equilateral triangles are shown instead, which are simply cross-sections of the regular tetrahedron. This leads to three parameters that can vary while the fourth is kept constant, and three crosssections are plotted to show a representative evolution of the response along the design space.

In Figure 4, the fixed CNF content is different from top to bottom triangles, 0,5 and $10 \% \mathrm{w}$ respectively. Empty zones in the diagram in top and middle rows are out of the DoE's boundaries. Additionally, a point in a triangle describes one formulation. The further a point is from a corner, the lower the content attributed to that corner is, e.g. the formulation described at the bottom left angle has the highest possible C65 content, equal to the value written there. Conversely, if a point is on the right edge, C65 is worth $0 \%$ w. Finally, the sum of all components is always equal to $100 \%$ w, i.e. when one component's content increases, the other three contents must decrease. For a better visualization, all triangles are the same size, even though they are smaller the lower the row, as described by the regular tetrahedron on the left. For instance, the top triangles have a height of $22.5 \%$ w whereas the bottom ones' height are $12.5 \%$ w. 
Quick guide to coded variables

An example of coded equation can be found below, Equation S1.

$$
Q_{15 C}(L T O \cdot T P E)^{0.7}=18-3 \times N m+5 \times \text { EleCond }-9 \times \text { Thickness }
$$

For minimum $\mathrm{Nm}$ and thickness and maximum electronic conductivity, each factor will then be equal to $-1,-1$ and +1 respectively, and the capacity would be worth $161 \mathrm{mAh} \cdot \mathrm{g}^{-1}$. Evidently, such an electrode had not been made because reaching $120 \mathrm{~S} \cdot \mathrm{m}^{-1}$ would require a very high conductive additives fraction, thus making the electrode thicker. Finally, coded variables allow to compare the relative impact of different factors. For instance, at $15 \mathrm{C}$, the electronic conductivity of LTOTPE electrodes was responsible for $30 \%$ of their capacity. The positive sign of the coefficient further indicates that an increase in electronic conductivity would result in an increase of capacity. 

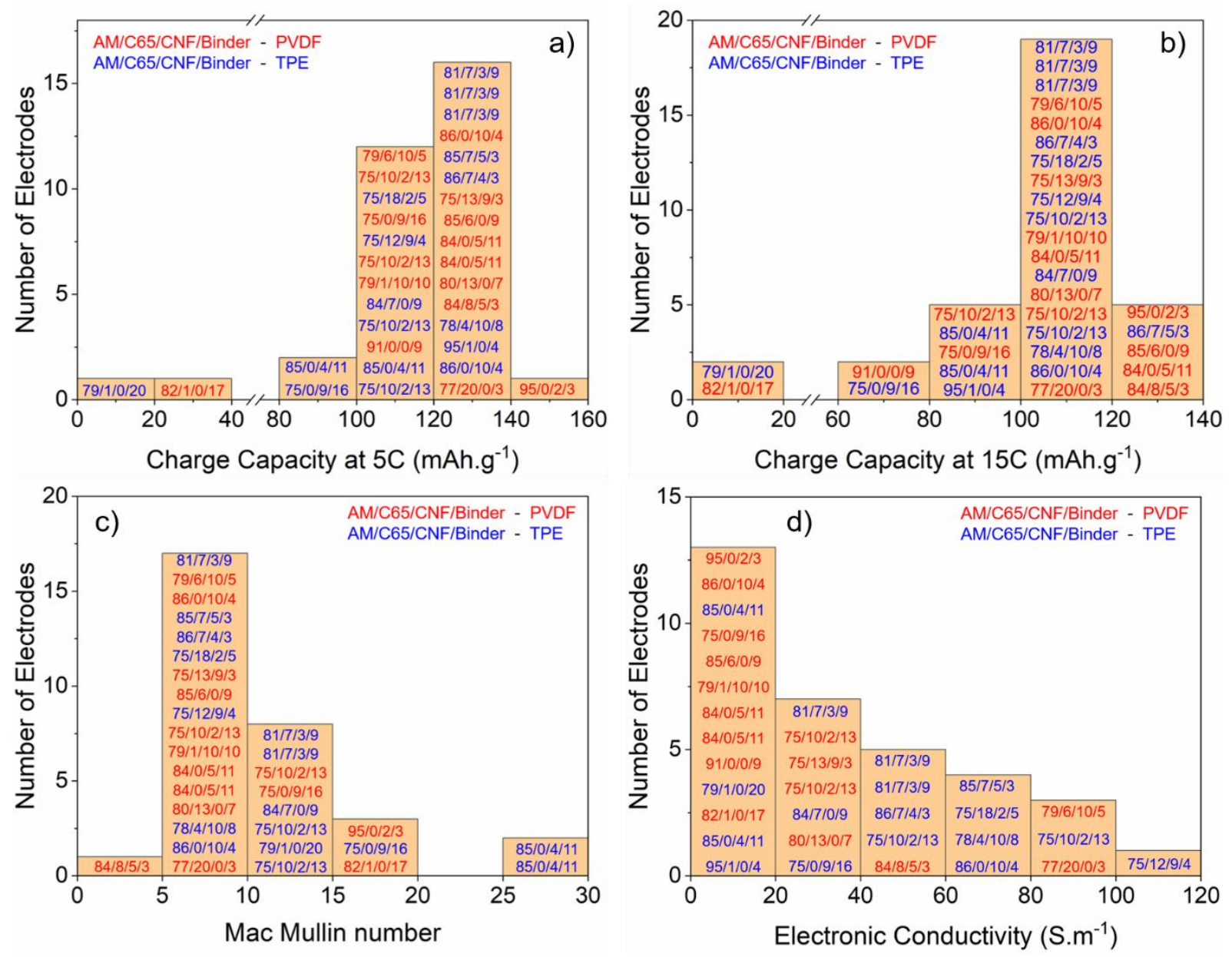

Figure S1. Data distribution for LTO-RUNs of different responses. Charge capacity at a) 5C and

b) $15 \mathrm{C}, \mathrm{c})$ Mac Mullin number and d) electronic conductivity 


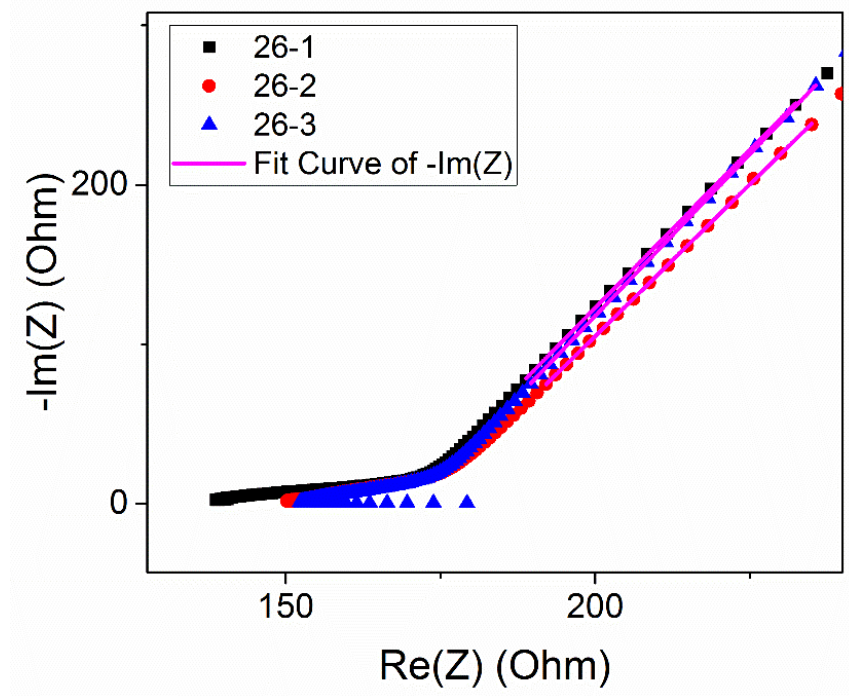

Figure S2. Electrochemical Impedance Spectroscopy measurement between $200 \mathrm{kHz}$ and 100 $\mathrm{mHz}$ with $20 \mathrm{mV}$ amplitude on a symmetrical cell to measure the tortuosity. The electrolyte is 10 mM TBAClO 4 in EC:DEC (w:w). Each curve represents one measurement carried out on one pair of electrodes for LFP-RUN26, the magenta single point is the smallest value of each RUN, R HFR, $_{\text {, }}$ and the magenta line is the linear fit used to obtain $\mathrm{Z}_{\mathrm{el}}$.

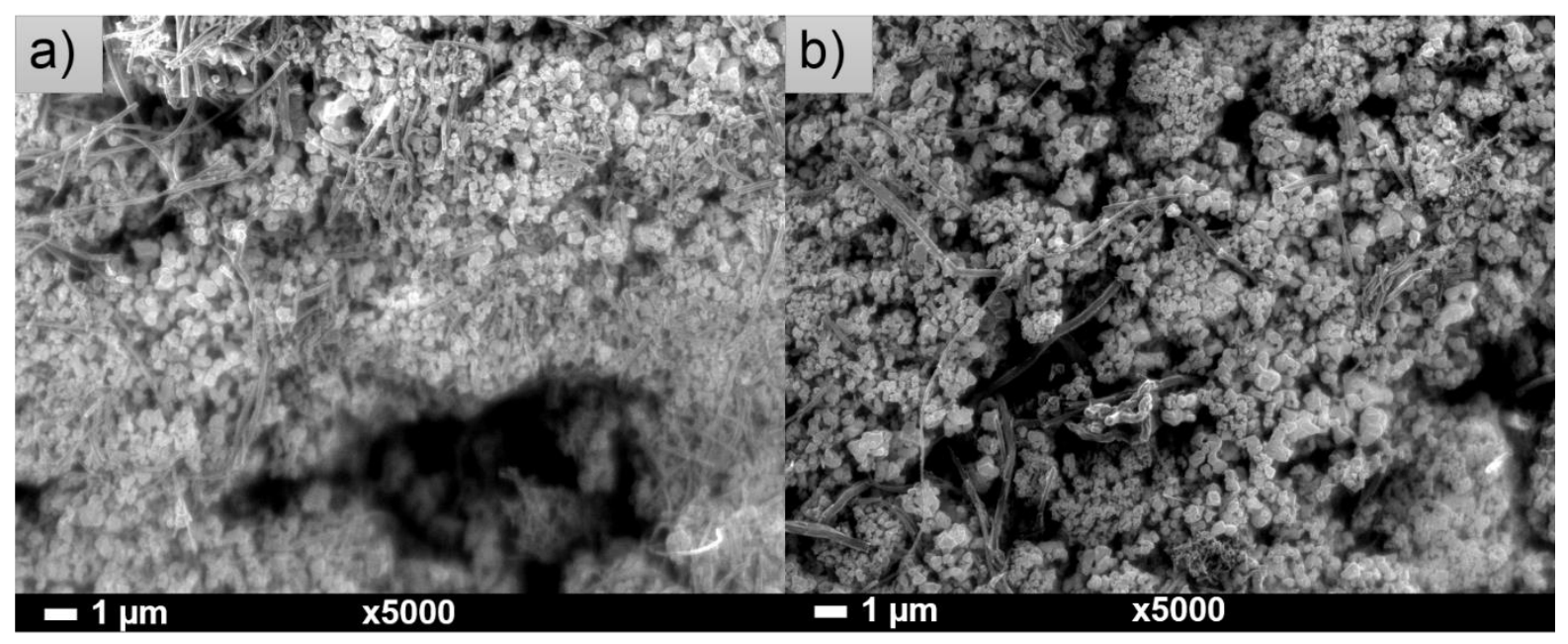

Figure S3. SEM micrographs of a) LTO-RUN02 and b) LTO-RUN27 magnified 5,000 times. The formulation is 86.5-0-10-3.5 (AM-C65-CNF-Binder). The difference between both formulations is binder type, a) Lotader 5500 and b) PVDF. 
Table S1. Some results of GCPL, tortuosity and electronic conductivity measurements for all LFP-

RUNs. Each value is the mean of 3 experiments. Blue lines represent optimized formulations.

\begin{tabular}{|c|c|c|c|c|c|c|c|c|c|c|c|}
\hline $\begin{array}{l}\text { LFP- } \\
\text { RUN }\end{array}$ & $\begin{array}{c}\mathbf{L F P} \\
\%_{\mathrm{w}}\end{array}$ & $\begin{array}{l}\mathrm{C65} \\
\%_{\mathrm{w}}\end{array}$ & $\begin{array}{c}\text { CNF } \\
\%_{\mathrm{w}}\end{array}$ & $\begin{array}{c}\text { Binder } \\
\%_{\mathrm{w}}\end{array}$ & $\begin{array}{c}\text { Binder } \\
\text { Type }\end{array}$ & $\begin{array}{c}\text { Q C/25 } \\
\mathrm{mAh}^{-} \mathbf{g}^{-1}\end{array}$ & $\begin{array}{c}\text { Q 5C } \\
\mathbf{m A h}^{\prime} \mathbf{g}^{-1}\end{array}$ & $\begin{array}{c}\text { Q 15C } \\
\mathrm{mAh}^{\prime} \mathrm{g}^{-1}\end{array}$ & $\mathrm{Nm}$ & $\begin{array}{c}\text { Electronic } \\
\text { Conductivity } \\
\mathbf{S} \cdot \mathbf{m}^{-1}\end{array}$ & $\begin{array}{c}\text { Thickness } \\
\mu \mathrm{m}\end{array}$ \\
\hline 1 & 75.0 & 0.0 & 9.5 & 15.6 & HNBR & 115.8 & 96.7 & 54.2 & 11.9 & 13.0 & 41.7 \\
\hline 2 & 75.1 & 10.2 & 3.6 & 11.1 & TPE & 118.0 & 93.9 & 59.8 & 6.7 & 72.8 & 47.3 \\
\hline 3 & 75.0 & 12.9 & 9.1 & 3.0 & HNBR & 116.3 & 98.5 & 77.6 & 7.2 & 40.4 & 43.7 \\
\hline 4 & 88.3 & 4.4 & 0.0 & 7.2 & PVDF & 140.0 & 124.9 & 106.1 & 6.4 & 1.5 & 31.7 \\
\hline 5 & 75.0 & 10.2 & 2.7 & 12.1 & HNBR & 117.9 & 99.7 & 80.8 & 12.6 & 12.4 & 49.0 \\
\hline 6 & 84.3 & 8.9 & 3.9 & 3.0 & HNBR & 130.9 & 114.2 & 96.2 & 6.1 & 39.9 & 34.7 \\
\hline 7 & 85.4 & 5.8 & 0.0 & 8.8 & HNBR & 134.4 & 114.3 & 86.8 & 11.4 & 4.6 & 35.7 \\
\hline 8 & 75.0 & 0.0 & 5.0 & 20.0 & PVDF & 115.5 & 98.2 & 47.5 & 17.2 & 6.4 & 37.0 \\
\hline 9 & 75.0 & 9.7 & 4.0 & 11.3 & PVDF & 117.5 & 102.9 & 84.9 & 7.0 & 17.5 & 44.3 \\
\hline 10 & 85.0 & 0.0 & 3.5 & 11.5 & PVDF & 133.8 & 116.2 & 95.1 & 9.2 & 7.2 & 29.7 \\
\hline 11 & 83.9 & 8.4 & 4.7 & 3.0 & TPE & 130.5 & 112.0 & 93.6 & 7.6 & 51.4 & 32.0 \\
\hline 12 & 89.0 & 0.0 & 8.0 & 3.0 & PVDF & 136.8 & 116.0 & 92.6 & 6.5 & 14.6 & 30.3 \\
\hline 13 & 77.0 & 20.0 & 0.0 & 3.1 & HNBR & 122.8 & 107.2 & 89.5 & 7.3 & 16.8 & 37.0 \\
\hline 14 & 85.6 & 0.0 & 3.0 & 11.5 & TPE & 130.8 & 89.3 & 23.9 & 20.4 & 11.4 & 35.7 \\
\hline 15 & 78.7 & 4.0 & 0.0 & 17.3 & PVDF & 122.7 & 107.6 & 86.3 & 12.0 & 4.0 & 32.0 \\
\hline 16 & 75.0 & 18.0 & 2.0 & 5.0 & TPE & 116.1 & 104.1 & 89.9 & 5.1 & 107.7 & 38.0 \\
\hline 17 & 86.0 & 0.0 & 3.0 & 11.1 & TPE & 133.8 & 94.1 & 21.2 & 26.9 & 12.7 & 31.7 \\
\hline 18 & 78.4 & 1.5 & 0.0 & 20.1 & HNBR & 118.3 & 19.9 & 7.8 & 10.2 & 0.5 & 30.0 \\
\hline 19 & 79.2 & 3.3 & 9.3 & 8.3 & TPE & 122.4 & 103.9 & 74.3 & 6.4 & 49.2 & 44.7 \\
\hline 20 & 77.3 & 2.7 & 0.0 & 20.0 & TPE & 25.9 & 0.0 & 0.0 & 1067 & 0.9 & 40.0 \\
\hline 21 & 85.0 & 0.0 & 3.5 & 11.5 & PVDF & 131.8 & 112.6 & 90.0 & 14.2 & 5.7 & 30.7 \\
\hline 22 & 77.4 & 1.9 & 10.0 & 10.7 & PVDF & 120.9 & 102.4 & 81.8 & 13.5 & 13.9 & 38.3 \\
\hline 23 & 75.0 & 10.2 & 2.7 & 12.0 & HNBR & 114.0 & 94.2 & 75.3 & 7.9 & 11.3 & 45.3 \\
\hline 24 & 94.9 & 0.0 & 0.0 & 5.1 & PVDF & 148.2 & 128.1 & 95.7 & 21.8 & 0.6 & 26.0 \\
\hline 25 & 84.1 & 0.0 & 4.6 & 11.3 & HNBR & 128.3 & 105.0 & 49.5 & 20.3 & 15.5 & 32.0 \\
\hline 26 & 94.9 & 2.0 & 0.0 & 3.1 & TPE & 146.6 & 126.6 & 104.7 & 8.0 & 5.8 & 26.3 \\
\hline 27 & 85.2 & 9.9 & 1.8 & 3.0 & PVDF & 134.2 & 116.2 & 85.8 & 7.4 & 6.8 & 29.7 \\
\hline 28 & 75.0 & 13.7 & 8.3 & 3.0 & PVDF & 119.2 & 102.2 & 86.1 & 8.1 & 16.0 & 41.0 \\
\hline 29 & 95.0 & 0.0 & 1.5 & 3.6 & HNBR & 144.4 & 120.2 & 94.7 & 11.0 & 1.4 & 29.7 \\
\hline 30 & 78.4 & 13.4 & 3.1 & 5.2 & TPE & 122.0 & 105.2 & 85.9 & 7.7 & 57.4 & 39.7 \\
\hline 31 & 75.0 & 20.0 & 0.0 & 5.0 & PVDF & 116.2 & 101.8 & 86.6 & 6.1 & 9.8 & 39.0 \\
\hline 32 & 84.1 & 0.0 & 4.6 & 11.3 & HNBR & 129.0 & 103.3 & 44.8 & 9.7 & 5.6 & 36.0 \\
\hline 33 & 82.7 & 8.8 & 0.0 & 8.6 & TPE & 126.4 & 99.9 & 62.4 & 6.1 & 42.0 & 38.0 \\
\hline 34 & 81.1 & 6.0 & 9.9 & 3.0 & PVDF & 125.9 & 108.7 & 92.0 & 7.8 & 17.3 & 40.7 \\
\hline 35 & 90.5 & 6.4 & 0.0 & 3.0 & HNBR & 138.8 & 117.0 & 88.5 & 9.0 & 7.2 & 30.7 \\
\hline 36 & 75.0 & 0.0 & 8.7 & 16.3 & TPE & 115.3 & 25.8 & 4.6 & 99.0 & 40.0 & 37.7 \\
\hline 37 & 87.0 & 0.0 & 10.0 & 3.0 & TPE & 134.1 & 117.2 & 98.4 & 7.3 & 54.0 & 35.7 \\
\hline 38 & 86.9 & 0.0 & 10.0 & 3.1 & HNBR & 134.2 & 114.0 & 85.7 & 8.0 & 16.8 & 35.7 \\
\hline 39 & 74.9 & 9.7 & 4.0 & 11.4 & PVDF & 115.6 & 98.2 & 80.9 & 6.3 & 4.2 & 37.3 \\
\hline 40 & 75.0 & 11.4 & 10.0 & 3.5 & TPE & 116.4 & 98.4 & 81.8 & 5.1 & 46.0 & 42.3 \\
\hline 41 & 81.2 & 6.7 & 3.4 & 8.7 & TPE & 125.7 & 104.6 & 78.1 & 7.4 & 51.1 & 40.3 \\
\hline 42 & 81.2 & 6.7 & 3.4 & 8.7 & TPE & 125.5 & 102.4 & 77.2 & 8.1 & 26.0 & 35.7 \\
\hline 43 & 81.2 & 6.7 & 3.4 & 8.7 & TPE & 124.6 & 103.4 & 77.2 & 8.7 & 31.3 & 35.3 \\
\hline 44 & 95.0 & 0.0 & 2.0 & 3.0 & TPE & 145.1 & 128.8 & 111.8 & 9.0 & 6.7 & 25.0 \\
\hline 45 & 95.0 & 1.0 & 1.0 & 3.0 & TPE & 146.0 & 126.8 & 111.8 & 14.5 & 6.6 & 25.0 \\
\hline 46 & 95.0 & 0.0 & 0.0 & 5.0 & TPE & 147.1 & 129.4 & 107.6 & 8.8 & 0.8 & 25.0 \\
\hline
\end{tabular}


Table S2. Some results of GCPL, tortuosity and electronic conductivity measurements for all LTO-RUNs. Each value is the mean of 3 experiments. Blue lines represent optimized formulations.

\begin{tabular}{|c|c|c|c|c|c|c|c|c|c|c|c|}
\hline $\begin{array}{l}\text { LTO- } \\
\text { RUN }\end{array}$ & $\begin{array}{c}\text { LTO } \\
\%_{w}\end{array}$ & $\begin{array}{l}\text { C65 } \\
\%_{w}\end{array}$ & $\begin{array}{c}\text { CNF } \\
\%_{w}\end{array}$ & $\begin{array}{c}\text { Binder } \\
\%_{w}\end{array}$ & $\begin{array}{l}\text { Binder } \\
\text { Type }\end{array}$ & $\begin{array}{c}\mathrm{Q} \mathrm{C} / 25 \\
\mathrm{mAh}^{\cdot} \mathrm{g}^{-1}\end{array}$ & $\begin{array}{c}\text { Q 5C } \\
\mathrm{mAh}^{\cdot} \mathrm{g}^{-1}\end{array}$ & $\begin{array}{c}\text { Q 15C } \\
\mathrm{mAh}^{\cdot} \mathrm{g}^{-1}\end{array}$ & $\mathbf{N m}$ & $\begin{array}{c}\text { Electronic } \\
\text { Conductivity } \\
\mathbf{S} \cdot \mathbf{m}^{-1}\end{array}$ & $\begin{array}{c}\text { Thickness } \\
\mu \mathrm{m}\end{array}$ \\
\hline 1 & 77.0 & 20.0 & 0.0 & 3.0 & PVDF & 130.8 & 124.3 & 79.1 & 8.9 & 89.0 & 34.7 \\
\hline 2 & 86.4 & 0.0 & 10.0 & 3.6 & TPE & 140.4 & 130.6 & 77.2 & 5.5 & 65.7 & 34.3 \\
\hline 3 & 95.0 & 0.6 & 0.0 & 4.5 & TPE & 152.9 & 120.7 & 71.3 & 0.0 & 0.0 & 28.0 \\
\hline 4 & 78.5 & 3.8 & 10.0 & 7.6 & TPE & 126.9 & 121.4 & 74.5 & 6.4 & 61.8 & 37.7 \\
\hline 5 & 84.2 & 8.1 & 4.7 & 3.0 & PVDF & 141.9 & 138.1 & 92.5 & 4.2 & 44.6 & 31.7 \\
\hline 6 & 75.0 & 10.0 & 2.5 & 12.5 & TPE & 121.2 & 114.4 & 66.2 & 10.8 & 81.8 & 37.7 \\
\hline 7 & 84.8 & 0.0 & 4.1 & 11.1 & TPE & 126.1 & 112.8 & 30.5 & 29.6 & 7.1 & 31.0 \\
\hline 8 & 81.9 & 0.6 & 0.0 & 17.5 & PVDF & 125.3 & 26.4 & 0.0 & 16.0 & 0.0 & 33.0 \\
\hline 9 & 78.9 & 1.1 & 0.0 & 20.0 & TPE & 31.6 & 0.0 & 0.0 & 12.6 & 0.0 & 44.3 \\
\hline 10 & 74.6 & 0.0 & 9.2 & 16.1 & TPE & 113.2 & 98.5 & 13.9 & 16.0 & 22.7 & 42.3 \\
\hline 11 & 90.6 & 0.0 & 0.0 & 9.4 & PVDF & 145.7 & 106.6 & 78.2 & 0.0 & 0.0 & 28.3 \\
\hline 12 & 74.9 & 10.1 & 2.4 & 12.6 & TPE & 125.1 & 113.6 & 47.7 & 10.1 & 55.6 & 37.0 \\
\hline 13 & 80.0 & 13.1 & 0.0 & 6.9 & PVDF & 133.6 & 126.4 & 81.5 & 7.7 & 27.5 & 33.3 \\
\hline 14 & 84.5 & 0.0 & 4.6 & 10.9 & PVDF & 135.2 & 128.2 & 87.5 & 9.3 & 6.5 & 31.0 \\
\hline 15 & 83.9 & 7.3 & 0.0 & 8.8 & TPE & 123.9 & 118.9 & 75.0 & 11.0 & 28.9 & 35.7 \\
\hline 16 & 84.4 & 0.0 & 4.6 & 11.0 & PVDF & 134.3 & 124.7 & 76.2 & 7.9 & 12.8 & 32.0 \\
\hline 17 & 79.4 & 0.9 & 10.0 & 9.7 & PVDF & 127.7 & 116.8 & 62.0 & 7.0 & 17.5 & 33.7 \\
\hline 18 & 75.0 & 9.9 & 2.5 & 12.6 & PVDF & 122.0 & 115.2 & 71.3 & 7.2 & 31.3 & 36.3 \\
\hline 19 & 75.0 & 12.2 & 9.1 & 3.7 & TPE & 122.6 & 110.0 & 77.1 & 6.7 & 118.6 & 38.3 \\
\hline 20 & 74.9 & 0.0 & 8.9 & 16.1 & PVDF & 113.6 & 102.7 & 61.0 & 10.1 & 17.0 & 35.3 \\
\hline 21 & 85.4 & 6.2 & 0.0 & 8.4 & PVDF & 140.6 & 131.2 & 79.8 & 6.7 & 12.0 & 28.7 \\
\hline 22 & 75.0 & 12.5 & 9.5 & 3.0 & PVDF & 129.2 & 126.0 & 69.9 & 6.4 & 39.0 & 40.7 \\
\hline 23 & 84.9 & 0.0 & 4.0 & 11.1 & TPE & 117.0 & 99.6 & 35.3 & 27.1 & 5.5 & 37.0 \\
\hline 24 & 75.0 & 18.0 & 2.0 & 5.0 & TPE & 121.3 & 115.0 & 82.2 & 6.7 & 77.0 & 39.3 \\
\hline 25 & 85.8 & 6.7 & 4.5 & 3.0 & TPE & 141.4 & 130.1 & 93.7 & 8.5 & 46.7 & 32.3 \\
\hline 26 & 85.8 & 6.7 & 4.5 & 3.0 & TPE & 140.4 & 130.9 & 86.8 & 9.4 & 70.1 & 32.7 \\
\hline 27 & 86.5 & 0.0 & 10.0 & 3.5 & PVDF & 140.5 & 132.7 & 85.6 & 10.0 & 17.1 & 30.0 \\
\hline 28 & 75.0 & 9.9 & 2.5 & 12.6 & PVDF & 122.8 & 112.7 & 67.4 & 11.5 & 36.7 & 38.0 \\
\hline 29 & 95.0 & 0.4 & 1.5 & 3.1 & PVDF & 154.6 & 149.3 & 104.7 & 16.0 & 3.5 & 26.3 \\
\hline 30 & 79.1 & 6.4 & 10.0 & 4.5 & PVDF & 129.8 & 119.9 & 83.6 & 7.0 & 86.3 & 35.3 \\
\hline 31 & 81.2 & 6.7 & 3.4 & 8.7 & TPE & 128.0 & 121.2 & 49.4 & 10.2 & 44.9 & 35.7 \\
\hline 32 & 81.2 & 6.7 & 3.4 & 8.7 & TPE & 131.3 & 121.8 & 27.5 & 11.4 & 41.0 & 36.0 \\
\hline 33 & 81.2 & 6.7 & 3.4 & 8.7 & TPE & 131.2 & 123.2 & 72.7 & 8.4 & 39.6 & 32.7 \\
\hline 34 & 95.0 & 0.0 & 2.0 & 3.0 & TPE & 147.5 & 131.5 & 95.9 & 8.5 & 1.0 & 28.0 \\
\hline 35 & 95.0 & 2.0 & 0.0 & 3.0 & TPE & 141.8 & 117.8 & 87.6 & 8.9 & 1.0 & 25.3 \\
\hline 36 & 95.0 & 1.0 & 1.0 & 5.0 & TPE & 153.7 & 142.7 & 103.0 & 0.0 & 0.0 & 25.3 \\
\hline
\end{tabular}

